\title{
Research on Methods of Human Eye Location
}

\section{Qingwen Ma ${ }^{a}$, Luqi Pan ${ }^{\mathrm{b}}$ and Fusheng Liu ${ }^{\mathrm{c}, *}$}

School of Mechanical and Vehicle Engineering, Linyi University, Linyi, People's Republic of China

a1335160476@qq.com, b1257034491@qq.com, ${ }^{\text {* }}$ liufusheng@lyu.edu.cn

Keywords: Eye location; Face recognition; Gabor transform; Image expansion

\begin{abstract}
Because of the poor anti-interference ability of the traditional eye location method, a new method of eye location based on Gabor transform is proposed in this study. The method firstly processes the image by Gabor filtering, and then expands the image so that the area of the face contour is connected. Finally, in order to display the human eye fully, secondary expansion is needed, so that the expanded human eye region can completely contain the human eye and present the original image and part of the human eye image. The experimentsl results show that this method can eliminate some interference regions and locate eyes accurately and effectively.
\end{abstract}

\section{Introduction}

The eye is an important organ of an individual. It is an important means for people to get outside information. People can perceive the world through their eyes. At the same time, the eye is also an important means of identity recognition, and the method that through the eye to determine the identity is less likely to be destroyed by external factors than fingerprint identification, and it can analyze identity accurately and steadily.

In addition, the eyes can reflect the rich psychological information of a man to a certain extent. For example, when people lie their eyes will twinkle, the eyes turn when they dream, and so on. As a result, the valuable information contained in the eye has been studied by more and more people, and the human eye localization and its function also obtains the extremely widespread application in reality.

In this paper, Matlab and the prior knowledge of image expansion are used to analyze and calculate the human eye area quickly and effectively ${ }^{[1]}$.

\section{Methods of Eye Location}

At present, a lot of eye location methods have been proposed, such as gray information method, geometry method, gradient projection method, symmetry method, Hof transform method,and so on. Each method has its own advantages and disadvantages ${ }^{[2,3,4]}$. There are some methods of the eye location as follows.

\section{Eye Location Based on Gray Information and Pupil Filter}

Eye location is generally divided into two steps: the first step is the initial location and the aim of this step is to locate the human eye roughly; the second step is accurate location and the aim of this step is to determine the exact position of the eyes with a specific method. A method of eye location based on projection histogram BP neural network. Firstly, the general position of human eye is detected from the given picture. Then, the position of the human eye is further analyzed by analyzing the projection histogram. After finding the approximate location of the human eye, the BP neural network is used to make the pupil filter work more accurately. Since the human face is first extracted from the image, then the exact position of the human eye is determined, so that the interference in the detected image except the face is excluded. Experimental results show that this method is especially effective in many ways when the background is complex and it can fundamentally solve the problem of locating eyes to eyebrows. 


\section{Method of Eye Location Based on Gray Histogram}

Using a more advanced method to calculate binary threshold of the face after the face is located. According to gray histogram information, the segmentation threshold can be obtained ${ }^{[4]}$. This method breaks through the traditional way that needs to make multiple calls to the two value threshold. After taking the two values of the eye area, the image is then processed by the processing of the image, and then the position of the human eye is determined according to the geometrical constraints. Then, according to the fixed position of the human eye on the human face, the two valued image of the face is processed and cut, simplified, and so on, thus reducing the amount of computation when the eyes are positioned. Finally, the geometrical constraint condition of the unified transform is used for the human eye, and the method of geometrical positioning is used to improve the accuracy of the image in different dimensions.

\section{Dynamic Face Detection Based on AdaBoost Algorithm and Human Eyes Location}

AdaBoost as a classifier algorithm, its basic idea is through the use of a specific method to make a large number of classification ability of weak classifiers work together, has a stronger classification ability of classifier ${ }^{[5]}$. So this method can rule out a lot of images that are not human faces and the detection speed is greatly improved. The detection ability of classifier has a great impact on the detection accuracy, although the detection speed is very fast, there is a certain rate of false detection. In order to avoid detecting the human face model falsely, a template matching algorithm is adopted.

\section{Eye Location Based on Skin Color and Template Matching}

A method of human eye location based on skin color and template matching is used to identify areas of the face by using the clustering of skin color. Compared with other gray features, the gray images of eyes and eyebrows are distinct, so it is better to locate the eye by using eye template ${ }^{[6]}$. The statistical template data can be obtained with a long and wide proportion of the eye images that contain the eyebrows. Human eye samples with a width ratio of 8 to 3 are extracted manually in 100 standard documents. Then, the eye samples are processed in a unified size, and their size is uniformly scaled to $64 * 24$ eyes samples, and the color images are transformed into gray images by gray scale calculation. Finally, the average eye template is obtained by averaging the 100 standard eye samples. The average eye template is obtained by a large number of sample images of the eye unified template. Template matching is performed in the standard window using the average eye template.

\section{The Human Eye Location Method Based on the Gabor Kernel Function}

\section{Theory of Gabor Kernel}

The definition of the Gabor transform kernel function is given, as shown in Eq. 1.

Where, $\mathrm{x}, \mathrm{y}$--Space coordinates of pixels

$\omega_{0}$--The radial center frequency of the frequency domain

$\theta$--direction

$\sigma$--Standard variance of Gauss function along $\mathrm{X}$ and $\mathrm{Y}$ directions;

The Gabor transform of a face image can be calculated using the convolution formula of the image and the Gabor transform kernel function, as shown in Eq. 2.

$$
C_{\varphi G}^{\left(\omega_{0}, \theta\right)}(x, y)=G(x, y) * * \varphi\left(x, y, \omega_{0}, \theta\right)
$$

Where, $C_{\varphi G}^{\left(\omega_{0}, \theta\right)}(x, y)$--The graph $\mathrm{G}(\mathrm{x}, \mathrm{y})$ corresponds to the radial center frequency is $\omega_{0}$. 


\section{Processing steps}

The processing steps of the eye location is given, as shown in Fig.1.

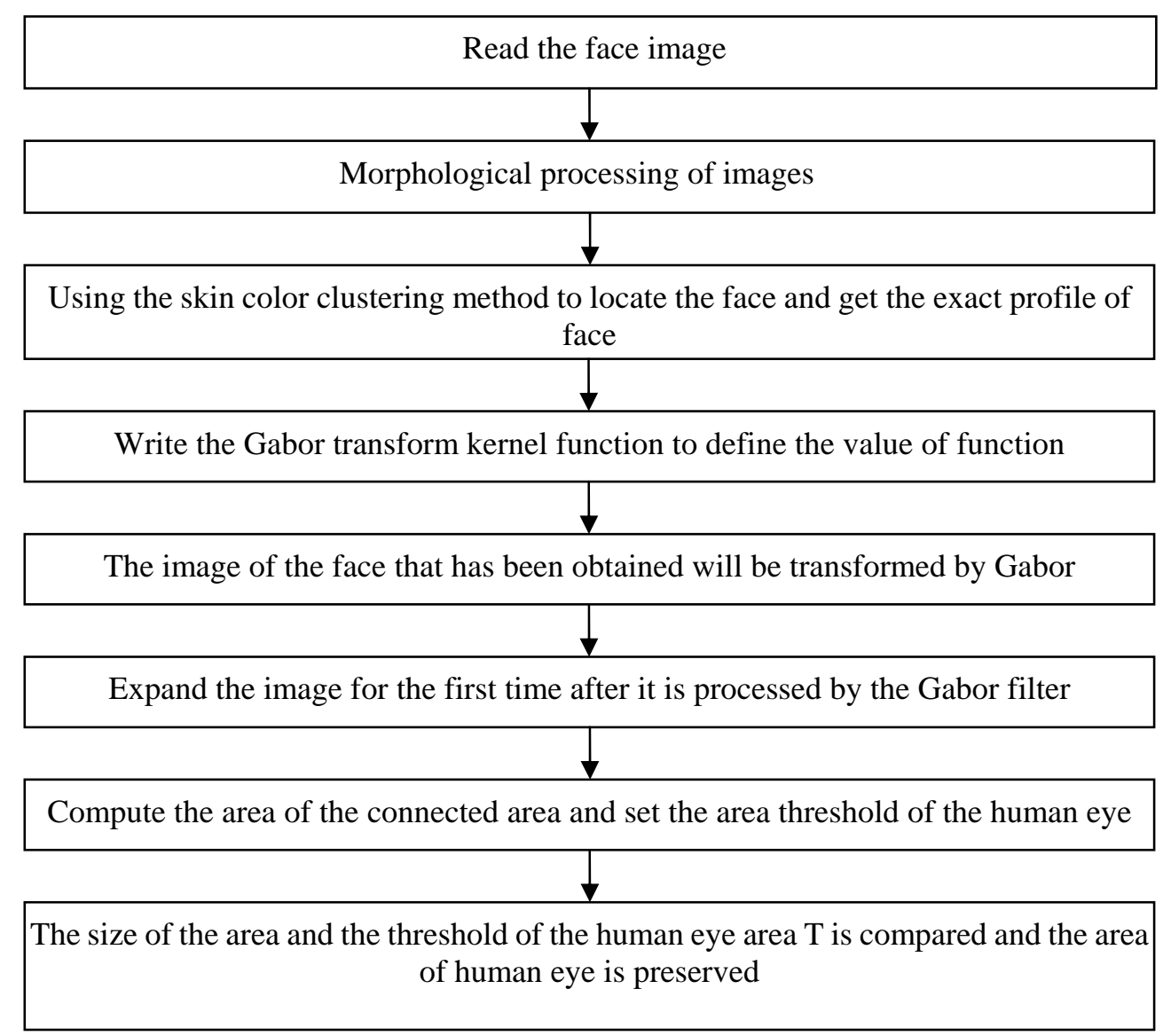

Fig.1 The flow chart of processing steps

Read the original image data and carry out the examination and determination of the face area by using the skin color clustering method. Locate the human eye with the Gabor transform function.

The face image that processed by Gabor filtering only reduces the range of the initial positioning of the human eye. So, the exact area of human eye needs to be extract after filtering processing. The white pixel area of the human eye area is small, and the white pixel area in the human eye area is separated from the rest. Other interference areas, such as the contours of the face, are larger in white pixels and the spacing between their white areas is small. The processed face images can be expanded to eliminate some interference regions and intercept the human eye region of interest.

First, the image of the faces is expanded, so that the area of the face contour is connected, and the area of the human eye region is independent of the other organ region. Then calculate the area of each connected area and set the threshold area of the human eye area T. When the area of a region is larger than the threshold T, it will be discarded, otherwise it is preserved as a large probability region of the human eye. Finally, because human the region of eye is small, second expansion must be carried out in order to make the eye emerge completely. The expanded human eye region can completely contain the human eye, and then presents the original image and the human eye image.

\section{Summary}

This paper gives a brief introduction to the methods of eye location, and compares the advantages with the disadvantages of various methods. Compared with the other methods, this method uses the prior knowledge of image expansion and Matlab to analyze and calculate the human eye area quickly and effectively. The experimental results show that this method can eliminate some interference regions and locate eyes accurately and effectively. 


\section{Acknowledgement}

This research was financially supported by the National Undergraduate Training Programs for Innovation and Entrepreneurship (No. 201610452057), and the Science and Technology Planning Project of Higher Education of Shandong Province (No. J16LB69), the Doctoral Scientific Research Startup Foundation of Linyi University (No. LYDX2016BS081), People’s Republic of China.

\section{References}

[1] D. Yang,H. B. Zhao, Z. Long, Matlab image processing example, Tsinghua university press, Beijing,2013.

[2] P. Xu, G. TONG, J. Qu, Face Detection in Video Based on Ada Boost Algorithm and Eye Location. Video Engineering. 35 (2011) 115 124

[3] Q. Y. Yang, Research and Application of Eye Localization Technology, South China University of Technology, Guangzhou, 2015.

[4] Y. Wu, Y. Yang, L. P. Wang, An Eye Location Algorithm Based on the Gray Information and the Pupil Filter, Computer Engineering and Application. 33 (2005) 45 47

[5] S. Li, D. H. Liu, L. S. Shen, Eye location Using Gar Transform, Measurement and Control Technology. 25 (2006) 27 32

[6] X. C. Dong, M. Shu, Eye location based on skin color and template matching, Journal of Optoelectronics . Laser. 45(2009) 237 239 